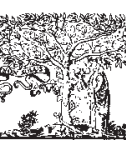

ELSEVIER

\title{
Use of Intraperitoneal ePTFE Gore Dual-Mesh Plus in a Giant Incisional Hernia After Kidney Transplantation: A Case Report
}

\author{
A.I. Lo Monte, G. Damiano, C. Maione, M.C. Gioviale, C. Lombardo, G. Buscemi, and M. Romano
}

\begin{abstract}
We evaluated the incidence of and predisposing factors for an incisional hernia after kidney transplantation. Numerous techniques have been used to repair postoperative fascial dehiscences or simple incisional hernias, but no clear treatment exists for giant hernias. Our aim was to obtain (1) a safe procedure to repair a large abdominal defect and reinforce the surrounding, fragile zones and (2) a simple, rapid technique to reduce the operative time. Herein we have described the surgical repair of a giant incisional hernia using intraperitoneal Gore ePTFE dual-mesh plus (Gore-Tex; W. L. Gore, Flagstaff, Ariz, USA) in a 55-year-old man status-post renal transplantation. Total necrosis of distal graft ureter had caused a giant urinoma. The patient was reexplored on day 2 posttransplantation with a primary fascial approximation. Thirty days after transplantation we discovered a large incisional hernia and performed a repair. No drain was used. The patient continued immunosuppressive therapy (cyclosporine, mycophenolate mofetil, prednisolone) and was discharged on postoperative day 4 with no complications. An ultrasonographic follow-up at 1 year revealed the prosthesis to be correctely positioned. Incisional hernia is not rare after renal transplantation but the real incidence is unknown. Immunosuppressive therapy, prolonged pretransplantation dialysis, obesity, and diabetes are probably the major causes of incisional hernias in these patients. Surgical complications of renal transplantation surgery, such as wound hematoma, urinoma, and lymphocele, are the most important predisposing factors for an incisional hernia. The use of intraperitoneal ePTFE dual-mesh is feasible, safe, and easy to repair a large incisional hernia in a kidney transplant patient.
\end{abstract}

W OUND PROBLEMS are infrequent complications following renal transplantation. The overall incidence of an incisional hernia after abdominal operations is $2 \%$ to $13 \%$. Among 2013 adult renal transplant recipients, $4.8 \%$ developed superficial or deep wound infections and $3.6 \%$ a fascial dehiscence or incisional hernia. ${ }^{1}$ Risk factors for these complications include obesity, reintervention through the same incision, immunosuppression, wound hematomas, urinoma, and lymphoceles. ${ }^{2,3}$ Numerous techniques have been used to repair postoperative fascial dehiscences and incisional hernias. Simple, noninfected incisional hernias in renal transplant patients may be repaired either primarily or with polypropylene mesh with good results. ${ }^{4-9}$ Large fascial dehiscences requiring abdominal reconstruction are rarely discussed in the transplant literature, but have been reported in nontransplant patients. ${ }^{10}$ The use of splitthickness skin grafts to cover exposed intraabdominal viscera with delayed repair of the abdominal defect with prosthetic mesh is well described in the general surgery literature. Single-stage abdominal wall reconstruction has also been accomplished using pedicled muscle, musculocutaneous, and fasciocutaneous flaps. Free tensor fascia lata (TFL) has also been employed with numerous advantages for abdominal wall reconstruction. ${ }^{11-14}$ Moreover, good results have been obtained with a mesh called Surgisis (Cook Surgical, Bloomington, Ind, USA), a 4-ply bioactive prosthetic material made from porcine small intestinal submucosa (SIS). It has shown particular promise for abdominal wall

From the Departments of General Surgery, Urgency, and Organ Transplantation (A.I.L., C.M., M.C.G., C.L., G.B., M.R.) and Clinical Medicine and Emerging Pathologies (G.D.), University of Palermo, School of Medicine, Palermo, Italy.

Address reprint requests to Attilio Ignazio Lo Monte, Department of General Surgery, Urgency, and Organ Transplantation (GEN.UR.T.O.), University of Palermo, School of Medicine, Via del Vespro, 129, 90127 Palermo, Italy. E-mail: ailomonte@unipa.it

(C) 2009 by Elsevier Inc. All rights reserved. 360 Park Avenue South, New York, NY 10010-1710 
reconstruction in infected fields. ${ }^{15-17}$ It eventually becomes replaced by well-organized host tissues ${ }^{17}$ with marrow-derived cells populating the SIS scaffold. ${ }^{18}$ Recently, in the pediatric renal transplant field, porcine dermal collagen implants have been used to assist abdominal wall closures due to donor organ size discrepancy. ${ }^{19}$ A large abdominal wall dehiscence in a renal transplant patient jeopardizes the survival of the graft. Since there is no clear treatment for this group of patients, we developed an easy, rapid technique to employ in a renal transplant patient with a wide fascial dehiscence requiring an otherwise complex abdominal plastic reconstruction.

\section{CASE REPORT}

This 55-year-old male renal transplant recipient developed an incisional hernia probably due to an urinoma secondary to a necrosis of the distal graft ureter requiring urgent reintervention on day 2 posttransplantation. The wound was closed with primary fascial approximation. The patient was discharged on day 14 posttransplantation, but at 1 month follow-up we discovered a large incisional hernia that rapidly became monstrous (Fig 1a,b). Abdominal computed tomographic (CT) scan demonstrated migration of omentum and small bowel into the sac with a $11.6 \times 14.7 \mathrm{~cm}$ defect in the wall diameter (Fig 1c). The preoperative preparation
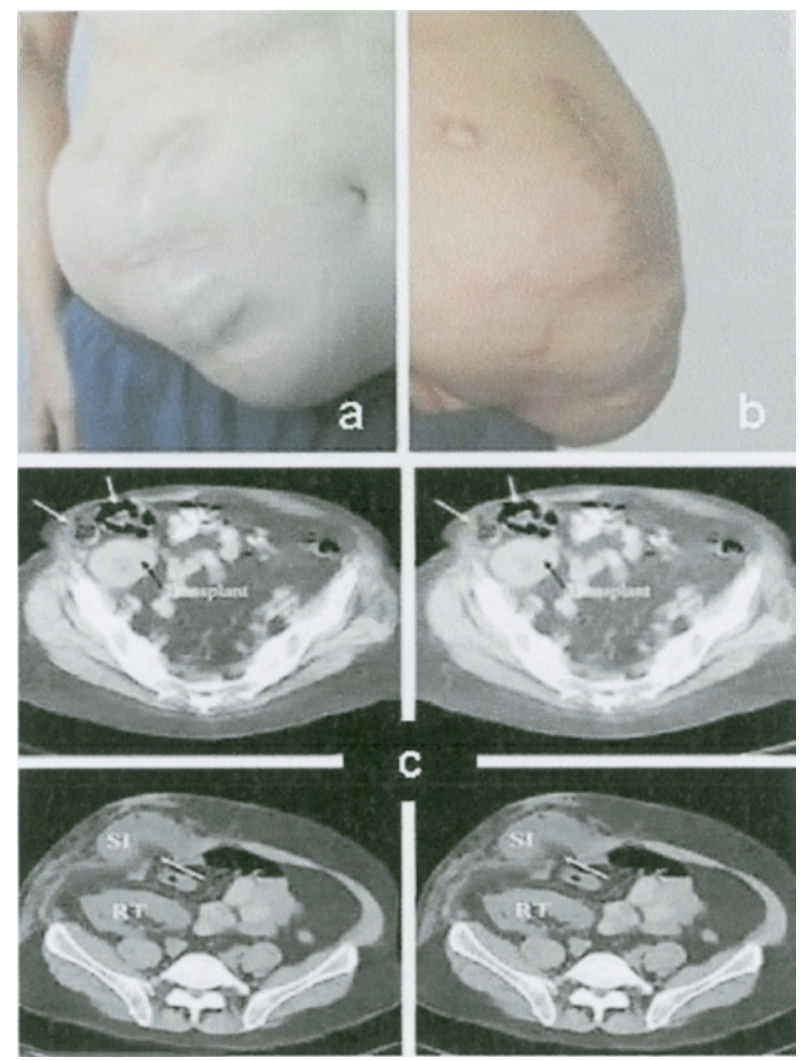

Fig 1. Frontal (a) and lateral (b) images of large incisional hernia in a 55-year-old male renal transplant patient. Abdominal CT scan (c) demonstrates migration of omentum and small bowel into the sac.
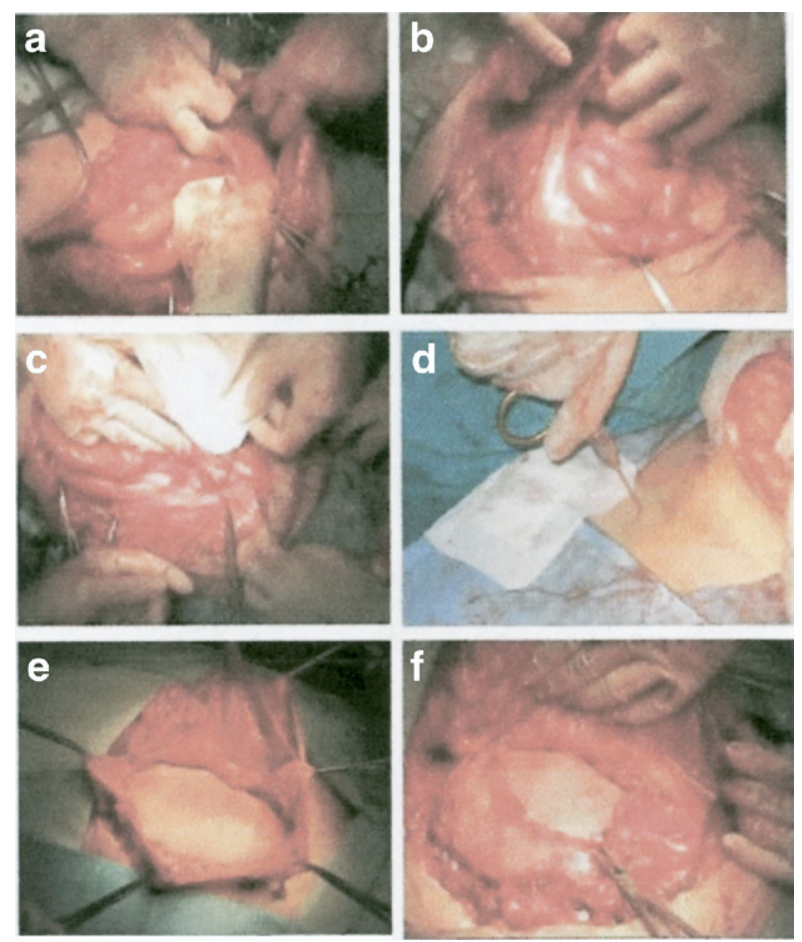

Fig 2. (a-f) Surgical procedure (see text for description).

of the patient started with remarkable weight loss. He was instructed in self-motivated respiratory physiotherapy.

The procedure for the repair of incisional hernia started with the excision of the scar. The herniated sac was opened carefully without dissection of surrounding subcutaneous tissue. Adhesions were gently removed from the internal sac surface and near the hernia ring. The dissection continued on the inside extending about $5 \mathrm{~cm}$ in all directions to allow safe intraperitoneal allocation of the mesh with a good overlapping of the hernia ring to reinforce the weak area near the abdominal wall defect. In fact, the quality of tissue adjacent to the parietal defect, muscle, and sheath is important for a strong attachment with the mesh. The expanded polytetrafluoroethylene (ePTFE) Gore dual-mesh plus (Gore-Tex; W. L. Gore, Flagstaff, Ariz, USA) advanced biomaterial contains 2 antimicrobial preservative agents, silver carbonate and chlorhexidine diacetate, which act to inhibit bacterial colonization and resist initial biofilm formation on the patch for up to 14 days postimplantation. For this reason, the prosthesis was anchored laterally inside to the inguinal ligament with 2-0 interrupted polypropylene stitches (Fig 2c) and laterally to the aponeurotic layer by a transparietal "stitch passer" (Fig 2d). The sutures were successively tied to the sheath muscular plane with little skin and subcutaneous tissue incised. The prosthesis was positioned without tension to the edges. The transparietal stitches were positioned along the prosthesis edge at a distance of about $5 \mathrm{~cm}$. The medial stitches only functioned to stretch the mesh on the internal abdominal layer. The other lateral edge was strongly attached to the inguinal ligament. Another continuous 2-0 polypropylene $360^{\circ}$ crown suture was then performed $2 \mathrm{~cm}$ inside the intraperitoneal hernial neck. Finally, the sac was fashioned from adjacent subcutaneous adherent tissue (Fig 2e) and sutured in the midline to isolate the prosthesis as far as possible from the skin wound, seeking to 
Fig 3. Frontal (a) and lateral (b) images of the abdomen at 1 year postsurgical treatment.
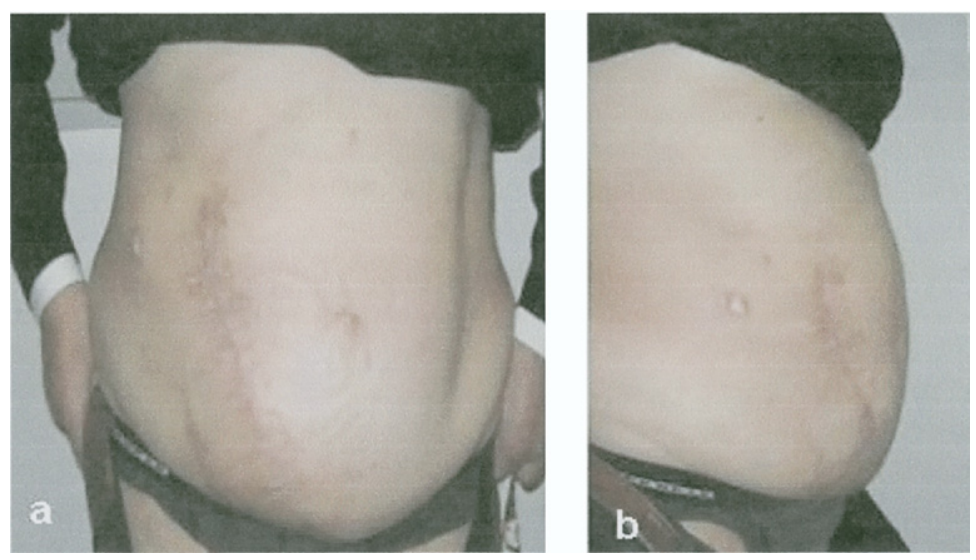

decrease the risk of infection (Fig 2f). Subcutaneous tissue and skin were closed without aspirative drains. Antibiotics were given as a prophylactic measure up to postoperative day 4. The patient continued immunosuppressive therapy (cyclosporine, mycophenolate mofetil, prednisolone) and was discharged on postoperative day 4 with no complications. At 1 year ultrasonographic follow-up, the prosthesis was positioned between the inguinal ligament and posterior abdominal wall without fluid collection or visceral adherence, an excellent functional result (Fig 3).

\section{DISCUSSION}

Incisional hernias are a prevalent complication among kidney allograft recipients, due to prolonged pretransplantation dialysis, immunosuppressive drugs, especially corticosteroids, and high prevalence of diabetes. In many renal transplant patients who have lower quadrant defects, classic surgical methods require extensive and difficult dissections and longer operative times. ${ }^{10-14}$ We chose this simple technique, which we successfully applied in an elderly patient with a giant incisional hernia, ${ }^{20}$ because it did not require dissection of intermediate layers, which engenders a long and invasive procedure associated with postoperative wound infection. Some authors have emphasized the risk of postoperative intestinal obstruction and bowel fistula in intraperitoneal positioning of the mesh, ${ }^{21,22}$ but the use of a hydrophobic material with reduced porosity (ePTFE) has minimized this complication. ${ }^{20,23}$ This straightforward technique involves minimal dissection and allows the aponeuroses, muscles, and subcutaneous tissues to remain intact. Proper positioning of the ePTFE prosthesis is extremely important; specifically, the material must be placed intraperitoneally so that it exceeds the hernia ring beyond $5 \mathrm{~cm}$. We also emphasize anchoring the prosthetic edges by transparietal stitches.

In conclusion, the simplicity of this "tension free" technique allowed an adequate repair without major aponeurosis dissection, thereby decreasing operative time. For this reason, our method to repair giant incisional hernias in kidney transplant patients fulfill's 3 conditions: (1) repairs the loss of abdominal wall without tension of opposite edges; (2) reestablishes the abdominal musculature inter- play; and (3) decreases operative time. Based on our experience in general surgery, ${ }^{20}$ this technique is feasible, safe, and easy. We therefore present this technical refinement as a potential solution to a challenging problem. Further follow-up is necessary to determine the long-term recurrence rate of this technique.

\section{REFERENCES}

1. Humar A, Ramcharan T, Denny R, et al: Are wound complications after a kidney transplant more common with modern immunosuppression? Transplantation 72:1920, 2001

2. National Report of Kidney Transplantation, JanuaryDecember 31, 2001. Available at: http://www.ustransplant.org

3. Knight RJ, Villa M, Laskey R, et al: Risk factors for impaired wound healing in sirolimus-treated renal transplant recipients. Clin Transplant 21:460, 2007

4. Mazzucchi E, Nahas WC, Antonopoulos I, et al: Incisional hernia and its repair with polypropylene mesh in renal transplant recipients. J Urol 160:816, 2001

5. Birolini C, Mazzucchi E, Utiyama EM, et al: Prosthetic repair of incisional hernia in kidney transplant patients. A technique with onlay polypropylene mesh. Hernia 5:31, 2001

6. Nguan CYC, Beasley KA, McAlister VC, et al: Treatment of renal transplant complications with a mesh hood fascial closure technique. Am J Surg 193:119, 2007

7. Antonopoulos IM, Nahas WC, Mazzucchi E, et al: Is polypropylene mesh safe and effective for repairing infected incisional hernia in renal transplant recipients? Urology 66:874, 2005

8. Mazzucchi E, Nahas WC, Antonopoulos I, et al: Incisional hernia and its repair with polypropylene mesh in renal transplant recipients. J Urol 166:816, 2001

9. Mahdavi R, Mehrabi M: Incisional hernia after renal transplantation and its repair with propylene mesh. Urol J 1:259, 2004

10. Vries Reilingh TS, van Goor H, Rosman C, et al: "Components separation technique" for the repair of large abdominal wall hernias. J Am Coll Surg 196:32, 2003

11. Feldt-Rasmussen K, Jensen O: Large ventral herniae treated with free fascial grafts: a follow-up study. Acta Chir Scand 111:403, 1956

12. McPeak CJ, Miller TF: Abdominal wall replacement. Surgery $47: 944,1960$

13. Disa JJ, Goldberg NH, Carlton JM, et al: Restoring abdominal wall integrity in contaminated tissue-deficient wounds using autologous fascia grafts. Plast Reconstr Surg 101:979, 1998

14. Fabian TC, Croce MA, Pritchard FE, et al: Planned ventral hernia: staged managements for acute abdominal wall defects. Ann Surg 219:651, 1994 
15. Ueno T, Pickett LC, de la Fuente SG, et al: Clinical application of porcine small intestinal submucosa in the management of infected or potentially contaminated abdominal defects. J Gastrointest Surg 8:109, 2004

16. Badylak S, Kokini K, Tullius B, et al: Morphologic study of small intestinal submucosa as a body wall repair device. J Surg Res 103:190, 2002

17. Badylak S, Park K, Peppas N, et al: Marrow derived cells populate scaffolds composed of xenogeneic extracellular matrix. Exp Hematol 29:1310, 2001

18. DeSagun EZ, Botts JL, Srivastava A, et al: Long-term outcome of xenogenic dermal matrix implantation in immunocompetent rats. J Surg Res 96:96, 2001

19. Pentlow A, Smart NJ, Richards SK, et al: The use of porcine dermal collagen implants in assisting abdominal wall closure of pediatric renal transplant recipients with donor size discrepancy. Pediatr Transplant 12:20, 2008

20. Lo Monte AI, Maione C, Moscato F, et al: Intraperitoneal mesh in elderly patients with large incisional hernias. A rapid and simple surgical treatment. Proceedings of 3rd International Congress of the Asia-Pacific Hernia Society, Singapore, October 25-27, 2007. Available at: http://www.aphs2007.com

21. Kaufman Z, Engelberg M, Zager M: Fecal fistula: a late complication of Marlex mesh repair. Dis Colon Rectum 24:543, 1981

22. De Guzman, Nyhus LM, Yared G, et al: Colocutaneous fistula formation following polypropylene mesh placement for repair of a ventral hernia: diagnosis by colonoscopy. Endoscopy 25:459, 1995

23. Maione C, Gambino G, Di Bona A, et al: PTFE mesh in renal allograft compartment syndrome. Transplant Proc 38:1049, 2006 